\title{
The consequences of contact with the criminal justice system for health in the transition to adulthood
}

\author{
Michael H. Esposito University of Washington, Seattle, USA \\ esposm2@uw.edu \\ Hedwig Lee \\ Margret T. Hicken \\ Lauren C. Porter \\ Jerald R. Herting \\ University of Washington, Seattle, USA \\ University of Michigan, Ann Arbor, USA \\ University of Maryland, College Park, USA \\ University of Washington, Seattle, USA
}

(Received February 2016 Revised July 2016)

http://dx.doi.org/10.14301/llcs.v8i1.405

\section{Abstract}

A rapidly growing literature has documented the adverse social, economic and, recently, health impacts of experiencing incarceration in the United States. Despite the insights that this work has provided in consistently documenting the deleterious effects of incarceration, little is known about the specific timing of criminal justice contact and early health consequences during the transition from adolescence to adulthood-a critical period in the life course, particularly for the development of poor health. Previous literature on the role of incarceration has also been hampered by the difficulties of parsing out the influence that incarceration exerts on health from the social and economic confounding forces that are linked to both criminal justice contact and health. This paper addresses these two gaps in the literature by examining the association between incarceration and health in the United States during the transition to adulthood, and by using an analytic approach that better isolates the association of incarceration with health from the multitude of confounders which could be alternatively driving this association. In this endeavor, we make use of variable-rich data from The National Longitudinal Study of Adolescent to Adult Health $(n=10,785)$ and a non-parametric Bayesian machine learning techniqueBayesian Additive Regression Trees. Our results suggest that the experience of incarceration at this stage of the life course increases the probability of depression, adversely affects the perception of general health status, but has no effect on the probability of developing hypertension in early adulthood. These findings signal that incarceration in emerging adulthood is an important stressor that can have immediate implications for mental and general health in early adulthood, and may help to explain long lasting implications incarceration has for health across the life course.

\section{Keywords}

Incarceration, population health, causal inference, machine learning 


\section{Introduction}

The United States (U.S.) currently has the highest incarceration rate in the world (Western, 2006). Indeed, recent estimates show that nearly 732 of every 100,000 adults $(1,352$ of every 100,000 men, and 126 of every 100,000 women) are in the U.S. correctional population, whereas only 510 of 100,000 adults are incarcerated in Cuba (the country of at least 500,000 people with the second highest incarceration rate), and only 233 of 100,000 individuals are incarcerated in Chile (the OECD country with the second highest incarceration rate after the U.S.) (Annie E. Casey Foundation, 2013; Glaze \& Parks, 2011; Walmsley, 2013). Perhaps more consequential is that the U.S. leads the world in the confinement of youth, with estimates showing that 336 of every 100,000 youth are held in confinement (Annie E. Casey Foundation, 2013; Glaze \& Parks, 2011). For reference, in South Africa (the country with the second highest youth incarceration rate in the world), only 69 of every 100,000 youth are held in confinement (Annie E. Casey Foundation, 2011; Hazel, 2008). Because of the high rate of incarceration across the U.S. population, many youths have experienced some form of contact with the criminal justice system, either through their own incarceration or through the incarceration of a family member.

Given the staggeringly high prevalence of incarceration in the U.S., researchers have turned towards investigating the impact that incarceration has on the lives of those who experience it. This work has shown that having been incarcerated adversely affects social and economic well-being along multiple dimensions. Indeed, research has shown that former inmates earn lower wages and face greater difficulties in securing employment than equivalent individuals who have never been incarcerated (Holzer, Raphael \& Stoll, 2004; Western, 2002). In addition, formerly incarcerated individuals are more likely to experience housing difficulties (Geller \& Curtis, 2011; Western, Kling \& Weiman, 2001), have reduced social standing (Schnittker \& Bacak, 2013), are more likely to experience marital dissolution (Lopoo \& Western, 2005), and struggle maintaining parenting roles (Turney \& Wildeman, 2013).
A relatively new body of work has begun to document that incarceration adversely affects mental and physical health. Current and former inmates are more likely to contract infectious diseases, have higher risk of death, and are more likely to develop either chronic physical or mental conditions than members of the general population (Golzari, Hunt \& Anoshiravani, 2006; Maruschak, 2010; Massoglia, 2008; Massoglia \& Pridemore, 2015). Second-degree contact with incarceration, typically defined as having a parent or other family member incarcerated, has also been shown to be predictive of poor mental and physical health in both childhood and adulthood (Lee, Wildeman, Wang, Matsuko \& Jackson, 2014; Roettger \& Boardman, 2012; Wildeman, Schnittker \& Turney, 2012). These adverse health effects are thought to be maintained by numerous mechanisms. For instance, recall that income, occupation and employment opportunities are all limited by experience of incarceration. As each of these factors are inputs to good health, their restriction by incarceration translates into worse health for former inmates (Mirowsky \& Ross 2003; Schnittker \& John, 2007). Beyond the adverse social and economic consequences of incarceration, the experience of having once been incarcerated is itself a chronic stressor that may directly affect mental and physical health through psychosocial and biological pathways and may also work indirectly to impact health through economic strains and deterioration in family functioning, particularly if introduced during critical periods in the life course (Massoglia, 2008; Pervanidou \& Chrousos, 2012; Roettger \& Boardman, 2012).

Despite the above insights, critical gaps still exist in our understanding of the link between incarceration and health. In this paper, we address two areas in which our current understanding of the relation between incarceration and health is limited. Specifically, we (1) estimate the relation between incarceration and health over a limited time span (during the transition to adulthood). Previous research that has examined the health consequences of incarceration has focused almost exclusively on health well into adulthood, overlooking the possibility that incarceration at young ages may have immediate 
effects on health. Then, (2) we also employ methods designed to better isolate the association of incarceration on health from the large array of confounders which could be alternatively driving this association. In this endeavor, we make use of variable-rich data from The National Longitudinal Study of Adolescent to Adult Health (Add Health) and a non-parametric Bayesian machine learning technique (i.e. Bayesian Additive Regression Trees or BART). This combination of rich data and robust statistical learning approach allows us to effectively control for a high-dimensional set of features that surround the association between previous incarceration and health, and obtain a less biased estimate of previous incarceration's effect on health during this important period in the life course.

\section{Background}

\section{Incarceration, health, and the transition from adolescence to adulthood}

To date, most empirical studies linking incarceration to health have examined the effect of incarceration at any point during the life course on health at mid-life or later (Massoglia, 2008; Schnittker \& John, 2007; Schnittker, Massoglia \& Uggen, 2012). Limited attention has been paid towards whether and how experiences of incarceration, specifically during the transition to adulthood, might serve to impact health relatively early in adulthood. This gap is problematic as life course theory suggests that the transition to adulthood (e.g. approximately at ages 15-30 years) is a critically important stage for the development of social and economic wellbeing, as well as good mental and physical health, and that the disruption at this stage may set in motion a lifetime of poor health, and increased exposure to other healthadverse conditions and behaviours (Alwin \& Wray, 2005; Ben-Shlomo \& Kuh; 2002; Cable, 2014). Indeed, life course epidemiology has enumerated the ways in which adverse social exposures (such as incarceration) can have important impacts on health. Beyond the direct biological stress impact of incarceration, we are informed by the chains of risk formulation, which indicate that early experience of incarceration sets in motion additional risk behaviour, or clusters of behaviours related to short-term and long-term health (Cable, 2014). Incarceration works through these mechanisms (see below) to promote negative health, beyond just the biological impact of the stress generated from having been incarcerated. While our task here is to simply identify the presence of an incarceration effect, and not to model these chains or pathways, it is important to highlight some of these mechanisms, and note that they inform our general approach.

The transition to adulthood is a critical period in the life course where individuals acquire the human capital skills and material resources needed to secure future resources, and develop productive social relationships (Settersten, Furstenberg \& Rumbaut, 2005). Incarceration during this critical period removes individuals from opportunities to develop these skills and secure other forms of social and material resources, potentially setting up a chain of risks both in terms of limiting protective resources and in terms of engaging in additional health risk behaviours (Ben-Shlomo \& Kuh, 2002). Instead of having the opportunity to obtain additional educational credentials, or develop social networks that might lead to marriage or job opportunities, for instance, incarcerated individuals are left sidelined both economically and socially (Pettit \& Western, 2004). Even when individuals return to the community from prison they face substantial barriers to re-building human and social capital due to factors such as employment discrimination, social stigma and isolation (Rose \& Clear, 1998; Western et al., 2001).

As many of the skills and resources that are fostered in the transition to adulthood can be used to protect health over the life course, the disruption of this critical period by incarceration is likely to result in worse health over the course of life. For instance, educational attainment is a strong determinate of multiple health outcomes in early, mid, and late adulthood. Adults with higher levels of educational attainment have a lower risk of mortality, are less likely to smoke, have better self-rated health, and have lower levels of depression and anxiety than their less educated counterparts (Cutler \& Lleras-Muney, 2006; Mirowsky \& Ross, 2003). Because incarceration during the transition to adulthood restricts one's 
ability to attain higher levels of education it may also serve to restrict one's ability to attain good health. Similarly, because marriage buffers against numerous adverse health outcomes (such as premature mortality, or the development of functional limitations), the restricted marriage opportunities that result from incarceration during the transition to adulthood may leave individuals less well equipped to protect against challenges to their health (Robards, Evandrou, Falkingham \& Vlachantoni, 2012).

The direct chronic stress (i.e. stress that results from repeated, and/or prolonged exposure to adverse events) brought about by being incarcerated at this life stage might also have both immediate, and long lasting health implications, as argued by a biological programing perspective (Ben-Shlomo \& Kuh, 2002). Stress can stem from multiple sources related to incarceration, including severed relationships with family and/or family instability, shame and stigma both during and postimprisonment, as well as financial strain stemming from fines and fees incurred in court and other monetary costs associated with imprisonment and reduced job opportunities post-imprisonment. Along with being immediately damaging to physical and or mental health, the elevated chronic stress generated from incarceration during the transition to adulthood can lead to increased exposure to other health degenerating factors, which themselves worsen health later in the life course. For example, a recent study found that young adults who experienced incarceration were more likely to engage in unhealthy, stress-inducing behaviours (e.g. smoking cigarettes or unhealthy binge eating) than their counterparts who had no prior experience with incarceration (Porter, 2014). This poor health behaviour can lead to immediate poor physical health, as well as poor health behaviours later into adulthood. Because poor health behaviours later into adulthood can beget poor adult health, the elevated chronic stress brought about by incarceration at this life stage could function to damage health far after incarceration has occurred (Haas, 2007; Hale, Bevilacqua \& Viner, 2015). Given the potentially severe, negative health consequences that incarceration during the transition to adulthood could have on long-term health, more work is needed to increase our understanding of the relationship between incarceration at this transitional stage and health at this specific point in early adulthood.

\section{Capturing or Estimating the Effect of Incarceration on Health}

The likelihood of coming into contact with the criminal justice system is not stochastic; individuals from marginalised groups are more at risk of being incarcerated than their more advantaged counterparts (Western \& Pettit, 2010). Similarly, the risk of poor health is not uniformly distributed across the U.S. population; many marginalised individuals are more at risk of experiencing chronic poor health and disease compared to their more advantaged peers (Phelan, Link, \& Tehranifar, 2010; Phelan \& Link, 2015; Williams, Mohammed, Leavell \& Collins, 2010).

These two points suggest that the fundamental problem in obtaining unbiased estimates of incarceration's health effect lies in the possibility that the health disadvantage that former inmates face is not a consequence of incarceration, but rather, is a reflection of inequalities in their pre-incarceration conditions (Schnittker et al., 2012). Parsing the degree to which prior incarceration influences health from the multitude of factors that occur before incarceration and health, and are associated with both incarceration and health (i.e. confound the association) is especially important. If incarceration is saddling individuals with poor health, policy interventions that address experiences during and post-incarceration would be likely to improve the health of former inmates. If instead, the poor health of previously incarcerated individuals is wholly a reflection of features that come before incarceration occurred, interventions that address incarceration and its consequences would do little to enhance the health of those who have experienced incarceration. We expect that both phenomena are operating. Criminal justice contact during the transition to adulthood likely has a lasting impact on health, but some of this association may be explained by the fact that marginalised populations have both higher risks of incarceration, and higher risks of poor health 
(Phelan \& Link, 2015). This means that the association between incarceration and health will remain sizable and significant, but not as strong once adjustments are made for confounding variables.

Identifying the health consequences of incarceration is difficult because incarceration does not lend itself to random assignment. To study the health consequences of prior incarceration, it is necessary to compare the health of individuals with and without a history of incarceration in observational data, where the processes of how they came to their level of health, and how they came to their incarceration history are largely unknown, ineffectively modelled, and yet highly intertwined (Schnittker \& John, 2007). Given that the best course of action for improving former inmates' health is dependent on the degree to which incarceration itself influences health, more research is needed which uses observational data to explore the potentially causal relationship between incarceration and health. Our innovation is to use a statistical learning technique that selects and flexibly models a subset of important confounders from a large array of candidates, to compare the health of individuals who had experienced incarceration, with their health had they never experienced incarceration, and obtain less biased approximations of incarceration's health influence. The method is likely superior to standard regression approaches that include relevant controls, or instrumental regression approaches where there are, at best, weak instruments available to isolate incarceration's specific effects.

\section{Data and methods}

\section{Analytical framework}

To estimate the effect that incarceration during the transition to adulthood has on health we would like to observe the following quantity:

$$
\frac{1}{\sum_{i}^{n} A_{i}} A_{i}\left(E\left(Y_{i} \mid A_{i}=1, C_{i}\right)-E\left(Y_{i} \mid A_{i}=0, C_{i}\right)\right)
$$

In Equation 1, $i$ indexes individuals ( 1 to $n$ ), $A$ is an indicator of whether an individual had previously been incarcerated (i.e. $A=1$ if a youth had been incarcerated before their health was measured, and $A$ $=0$ if otherwise), $Y$ gives an individual's health, and $C$ is a vector of each individual's confounders (Ho, Imai, King \& Stuart, 2007).

This quantity, or the average treatment effect on the treated (ATT), represents a comparison of scenarios. The left-hand side of the minus sign (i.e. $\left.E\left[Y_{i} \mid A_{i}=1, C_{i}\right]\right)$ gives the average level of health among a group of individuals (with $C$ preincarceration traits) who were observed to have experienced incarceration. The right-hand side of the equation (i.e. $E\left[Y_{i} \mid A_{i}=0, C_{i}\right]$ ) gives an estimate of the average health of the same group of individuals (with the same $C$ background traits) had they never experienced incarceration. Because individuals and their background characteristics are held constant in the scenarios being compared, the difference given by the ATT represents a counterfactual of how different the health of individuals who had experienced incarceration would be had they actually never experienced incarceration.

Note that a more complete $C$, and more accurately modeled Equation 1, leads to a less biased estimate of incarceration's influence on health; the more relevant confounding factors that are held constant across counterfactuals, and the more accurately modeled each counterfactual, the higher the probability is that the difference in $E\left[Y_{i} \mid A_{i}=1, C_{i}\right]$ and $E\left[Y_{i} \mid A_{i}=0, C_{i}\right]$ is attributable to a change in prior incarceration status. To minimize bias in our calculation of Equation 1, we use a rich data set (The National Longitudinal Study of Adolescent to Adult Health) to flesh out $C$, and a machine learning approach (Bayesian Additive Regression Trees) to accurately specify the models needed to estimate Equation 1's counterfactual states.

\section{Data: The National Longitudinal Study of Adolescent to Adult Health}

We used data from the National Longitudinal Study of Adolescent to Adult Health (Add Health), which is an ongoing nationally representative, school-based study of adolescents in grades 7 to 12 that began in 
1994 (Harris, et al., 2009). In a sample of 132 U.S. schools all students were given an in-school questionnaire. A sub-sample of 20,745 was chosen for separate in-home child and parent interviews. Add Health currently includes four waves of data: Waves 1 (W1: 1994-95); 2 (W2: 1996); 3 (W3: 200102); 4 (W4: 2007-08). In W4, respondents ranged in age from 24-32 years. For our purposes the utility of the Add Health data is two-fold. First, the Add Health data contain a relatively large sample of individuals across the life-stages that we are interested in (i.e. adolescence and early adulthood). Second, these data contain multiple detailed measures of social, economic, psychological, and health-related factors. The richness of Add Health's measures allows us to select a diverse array of features for our set of confounders $(C)$; as a more fully defined $C$ allows for a more accurate estimation of the health effects of incarceration, this latter feature makes Add Health invaluable to our efforts.

\section{Measurement}

\section{Analytical samples and prior incarceration}

Incarceration may occur at any point over the course of the study. To ensure that we only control for confounders that occur prior to incarceration (and thus avoid either controlling away incarceration's influence or inducing post-treatment bias'), we split the data into two analytical samples (defined below) based upon the timing of an individual's first instance of incarceration (Acharya, Blackwell \& Sen, 2015; Gelman \& Hill, 2007).

At W4, respondents were asked, How many times have you been in jail, prison, a juvenile detention center, or other correctional facility? If a respondent had been incarcerated, they were then asked, How old were you when you (first) went to jail, prison, juvenile detention center or other correctional facility? Using this information, in conjunction with each individual's age across the Add Health waves, allows us to sort individuals into two analytical samples: individuals who were first incarcerated after W1 and prior to W2 are sorted into Sample I, those who were first incarcerated after W3 and before W4 are included in Sample II, and those individuals who had never experienced incarceration are included in both samples as controls. While we do not have exact dates of incarceration, using age at first incarceration does allow us, with reasonable accuracy, to ensure that W1 covariates occurred prior to incarceration for Sample I members, and that W1 and W3 covariates occurred prior to incarceration for Sample II individuals. To illustrate this sorting process, an individual who reported that they were incarcerated at age 19, and was 19 years old between W1 and W2, would be sorted into Sample I. In both samples incarceration is defined as a binary variable, where a 1 indicates that an individual had been incarcerated, and a 0 indicates that an individual had never been incarcerated.

\section{Confounders (C)}

To define confounders for each analytical sample, we draw information from the Add Health waves that predate an individual's first instance of incarceration. For Sample I, this means that we only control for W1 covariates, and for Sample II, this means that we control for both W1 and W3 covariates. Specific measures are selected as confounders based on the literature of the social and economic associates of incarceration and health. For example, we include a variable measuring the degree to which an individual feels that they are connected to others at their school as: (1) social control theory suggests that low social attachment increases the probability of deviance/incarceration: (2) many health researchers report social connectedness as an associate of good health (Hawe \& Shiell, 2000; Hirschi, 1969). Likewise because level of educational attainment is positively associated with health, and negatively associated with risk of incarceration, we include a measure of highest level of educational attainment in our confounder set (Mirowsky \& Ross, 2003; Pettit \& Western, 2004).

In total we select 93 variables from W1 for our Sample I confounder set. For Sample II, our confounders consist of the 93 variables from W1, plus 36 additional covariates from W3. . iiii $^{\text {The confounders }}$ we selected fell into the following general categories: demographic characteristics (e.g. gender; race), prior health status and behaviours (e.g. body mass index (BMI) prior to incarceration; frequency of being in ill- 
health) engagement in risky behaviour (e.g. frequency of drug use; frequency of fights), social connectedness (e.g. degree of closeness to individuals at school; degree of closeness to individuals in neighborhood), disposition characteristics (e.g. selfperceptions of intelligence; belief in ability to solve problems "rationally"), parental characteristics (e.g. level of education, parental incarceration history), and contextual residential characteristics (e.g. crime rate in residential area; poverty rate in residential Census tract). A full list of the confounders used in our analysis can be found in the supplemental material.

\section{Health Outcomes ( $Y$ )}

For this paper we examine three health outcomes: (1) an indicator of cardiovascular health that is increasing in prevalence among young adults (i.e. hypertension or raised blood pressure) (Nguyen et al., 2011), (2) a measure of general health status (i.e. excellent/very good self-reported health), and (3) a measure of mental health status (i.e. depression). These measures of general health and mental health were chosen as they have been examined in the adult population in relationship to incarceration, and also capture general wellbeing (Diamond, Wang, Holzer, Thomas \& Cruse, 2001; Massoglia, 2008; Schnittker \& John, 2007; Schnittker et al., 2012). Hypertension was chosen so that we may examine whether incarceration results in the immediate emergence of physiological problems that typically take more time to emerge. All health measures are obtained from W4.

In Add Health systolic and diastolic blood pressure (SBP, DBP) were recorded three times for each individual. As an estimate of true SBP and DPB, Add Health averaged the second and third reading of blood pressure. From this, hypertension was classified according to guidelines from the American Heart Association: individuals who had an average SBP greater than or equal to $140 \mathrm{mmHg}$, or an average DBP of at least $90 \mathrm{mmHg}$ were classified as having hypertension (Calhoun et al., 2009). Individuals who had been told by a doctor that they had high blood pressure or hypertension, or were taking hypertension medication were also classified as having hypertension. ${ }^{\text {iv }}$

Next, excellent/very good self-rated health was derived from a question asking respondents, "in general, how is your health?" Available responses were, excellent, very good, good, fair, or poor. Individuals who responded with either excellent or very good were grouped into one category with the reference group including those who reported good, fair or poor health. The decision to divide self-rated health along the aforementioned lines was based on two factors. First, exploratory analysis showed that larger disparities existed in the higher categories of self-rated health, rather than the more commonly examined "poor" or "fair" dichotomization of selfrated health. This is particularly so for younger populations who are generally healthy and have yet to develop chronic diseases. This categorization is consistent with other inquires that have examined self-rated health among children and young adults (e.g. Braveman \& Gottlieb, 2014; Mandal, Edelstein, Ma \& Minkovitz, 2013).

Finally, our depression measure is a nine-item version of the Center for Epidemiological Studies depression scale (CES-D) (Eaton, Muntaner, Smith, Tien \& Ybarra, 2004). Respondents were asked, on a scale from 0 to 3 (with 0 being never or rarely, and 3 being most of the time or all of the time) if they felt the following in the past seven days: you were bothered by things that usually don't bother you; you could not shake off the blues, even with help from your family and your friends; you felt that you were just as good as other people (for which the scale was reversed); you had trouble keeping your mind on what you were doing; you were depressed; you were too tired to do things; you enjoyed life (also reversed); you were sad; you felt that people disliked you (Cronbach's $\alpha=0.80$ ) (Boardman \& Alexander, 2011). The results from each of these questions were summed, and [following the advice of Boardman \& Alexander, 2011)], we classified any individual who scored over 10 as having depression. Note that our definition of depression is based on depressive symptomatology/states, and thus is not a clinical measure of depression. However, the CES-D depression scale is good measure of risk of developing clinical depression or anxiety disorders (Perreira, DeebSossa, Harris \& Bollen, 2005). For parsimony when we use 
the term depression in results, we mean depressive symptoms, in particular a depressive symptoms score of over 10 .

\section{Analysis: Bayesian Additive Regression Trees}

Estimating the ATT is conceptually straightforward. To calculate this quantity we simply: 1 ) regress the health outcome of interest $(Y)$ on an indicator of incarceration $(A)$ and the set of confounders chosen from Add Health (C), 2) use the resulting model to predict $E\left[Y_{i} \mid A_{i}=1, C_{i}\right]$ and $E\left[Y_{i} \mid A_{i}=0, C_{i}\right]$, and 3 ) record the difference between the two counterfactual states. In practice, however, this process is made difficult by the dimensionality of (or the number of variables in) the confounder set $C$, as well as the specification of these variables in the model (Ho et al., 2007).

To expand, recall that defining $C$ as a rich, robust vector of potential confounders is theoretically desirable; as Hill, Weiss and Zhai (2011) state, "the richer the pre-treatment information we can condition on, the more we may be willing to believe that [the ATT represents a real treatment effect]." While advantageous in this manner, working with a large set of confounders to define the ATT comes with a number of empirical challenges. For instance, modeling a large number of confounders can be accompanied by an array of computational problems. Traditionally employed models, such as linear or logistic regression, often perform poorly (e.g. they provide nonsensical/unstable parameter estimates), or even fail to converge when used to describe the relationship between an outcome and a highdimensional set of covariates (Hill et al., 2011). ${ }^{v}$

Moreover, model specification (or the process of deciding how covariates should be included in the model) is a major concern when working with a large set of potential confounders. Properly specified models are desirable as they lead to more accurate (i.e. less likely to mistake random noise in the data for real structure, or vice-versa) representations of reality than do their poorly specified counterparts (Green \& Kern, 2012; Hill, 2011), but the probability of incorrectly specifying a model scales with the number of covariates under consideration. That is, with every additional predictor included in the model comes an increase in the number of models that could possibly be fitted, and with an increase in the number of models that could possibly be fitted comes a higher risk of choosing a model that is poorly specified (Ho et al., 2007).

Adjudicating between specifications of highdimensional data can be a daunting task. Relying on prior knowledge to narrow the range of plausible model specifications is useful, but is often insufficient on its own (i.e. prior literature is often informative enough to allow researchers to determine which factors ought to be considered as potential confounders, but not informative enough to describe the appropriate functional relationships between said predictors) (Ho et al., 2007). ${ }^{\text {vi }}$ Even relying on principled model selection methods in conjunction with prior knowledge to adjudicate between models can be infeasible in high-dimensions. Approaches that search among plausible specifications for the "best fitting model" (such as stepwise or subset selection approaches) begin to become impracticable in the face of a large number of potential confounders because of the sheer number of specifications that exist in such a scenario (Green \& Kern, 2012; Ho et al., 2007; James, Witten, Hastie \& Tibshirani, 2015).

To alleviate the practical concerns that come with examining a high-dimensional space, we use Bayesian Additive Regression Trees (BART) to generate the model needed to estimate the ATT (Chipman, George \& McCulloch, 2010). Through an iterative process this nonparametric algorithm uses an ensemble of small regression-trees (i.e. regressions that model only a very small subset of confounding variables at one time) to select a subset of variables and their specifications, and produce an accurate yet generalizable model representation of the data (Hill, 2011). That BART is able to produce stable, coherent estimates in face of a (very) large number of covariates, and is adept at letting the data decide upon a model specification (that strikes a balance between accuracy and parsimony) means that we are able to largely avoid the computational and specification challenges that often accompany modeling a large set of covariates. Thus, our machinelearning approach increases our confidence that our models account for confounders in a way that makes 
the ATT representative of a change in health due to incarceration history.

\section{Results}

Because the number of variables used in our analysis is large, we do not present a full table of descriptive statistics in the body of the paper (that information can be found here: http://bit.ly/2ac14gi). Instead, to contextualise our analytical samples, we provide summary statistics on a handful of demographic covariates in table 1 :

\section{Table 1: Selected demographic statistics for analytical samples}

\begin{tabular}{cccc} 
& \multicolumn{1}{c}{ Incarcerated Individuals } & \\
$\begin{array}{c}\text { Statistic } \\
\begin{array}{c}\text { Mean age of first } \\
\text { incarceration }\end{array}\end{array}$ & 18.6 & $\begin{array}{c}\text { Sample II } \\
(n=586)\end{array}$ & $\begin{array}{c}\text { Never Incarcerated } \\
(n=9,399)\end{array}$ \\
W4 Mean Age & 28.6 & 25.2 & - \\
Percent White & 52 & 28.03 & 28.31 \\
Percent Black & 24 & 49 & 57 \\
Percent Male & 74 & 29 & 20 \\
Percent Born in US & 96 & 64 & 59 \\
$\begin{array}{c}\text { Percent with mother with a } \\
\text { college degree }\end{array}$ & 16 & 97 & 93 \\
Median Household Income & & 17 & 27 \\
(of Census Block Group) & 26,370 & 26,635 & 28,853
\end{tabular}

From table 1 we see that the average age of first incarceration in Sample I is 18.6 years. The mean age of first incarceration for Sample II (25.2 years) is somewhat late into the transition to adulthood. At Wave 4, where our chosen health outcomes were recorded, the mean age for all groups was approximately 28 years. Males and people of colour made up a larger proportion of the sample of former inmates than they did the sample of never incarcerated individuals. Moreover, it appears as if formerly incarcerated individuals were from more marginalised socioeconomic groups than individuals who had never been incarcerated; for instance, the proportion of never-incarcerated individuals who had a mother with a college degree was approximately $10 \%$ higher than the proportion of former inmates who similarly educated mothers.

Next, we examine how the health outcomes chosen from W4 vary by incarceration history. Figure 1 plots the difference in the proportion of previously incarcerated individuals with each health outcome and the proportion of never incarcerated individuals with each health outcome: 


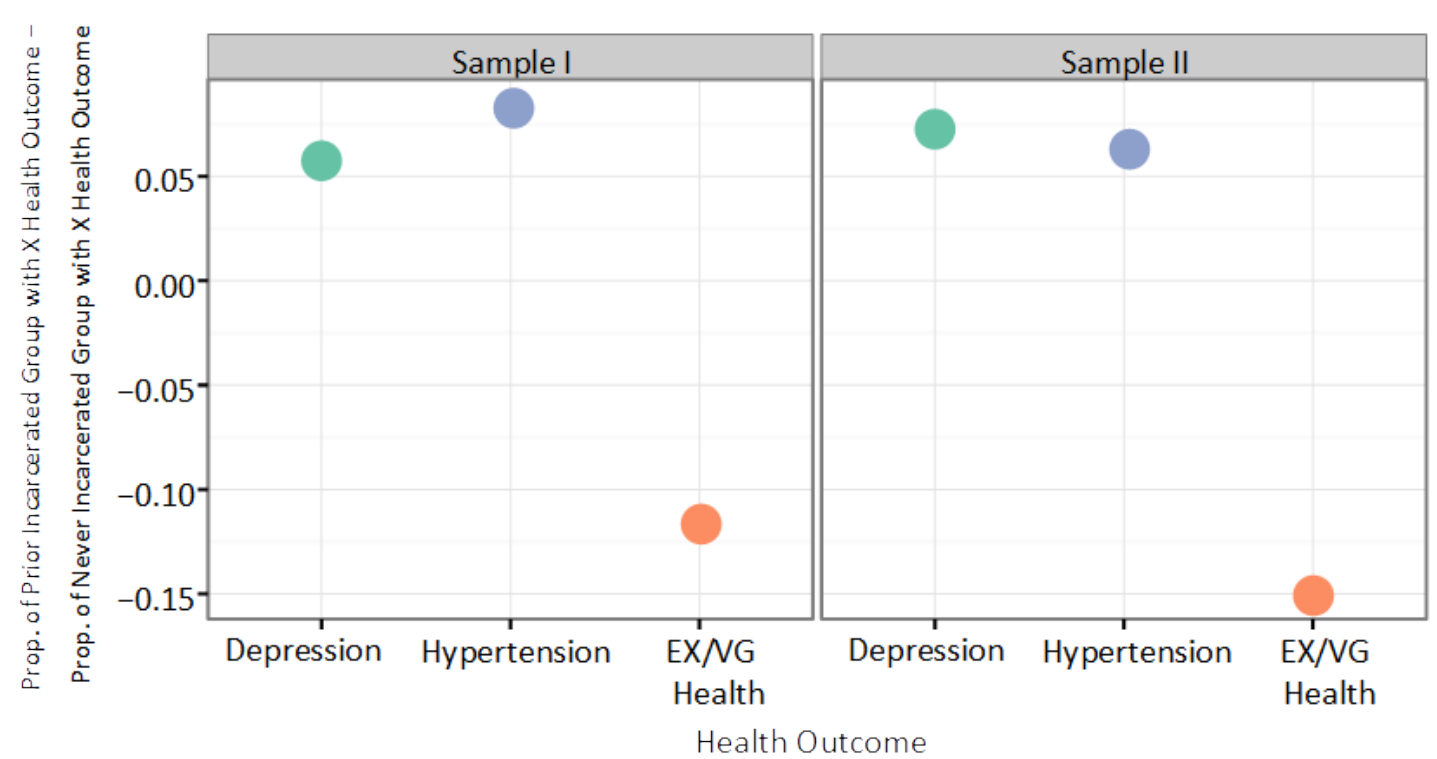

\section{Figure 1: Difference in the proportion of previously incarcerated individuals with each health outcome and the proportion of never incarcerated individuals with each health outcome. Note that EX/VG Health indicates excellent/very good health.}

As we can see from figure 1 , individuals with a history of incarceration were in worse health than their counterparts who had never been incarcerated. In Sample I, individuals who had been incarcerated were $6 \%$ more likely to suffer from depression, 12 points less likely to report being in excellent or very good health, and 8 points more likely to have hypertension than their peers with no history of incarceration. The inequalities in Sample II follow a similar pattern; individuals who were incarcerated between W3 and W4 were $8 \%$ more likely to be depressed, $15 \%$ less likely to report excellent or very good health, and $7 \%$ more likely to have hypertension than their counterparts who had never been incarcerated.

To examine if the health inequalities between previously incarcerated and never incarcerated individuals was a product of incarceration rather than a product of features that occur prior to incarceration, we estimate Equation 1 using the BART methodology described above. vii Figure 2 plots the estimated ATTs (and their 95\% confidence intervals) recovered from our models: 


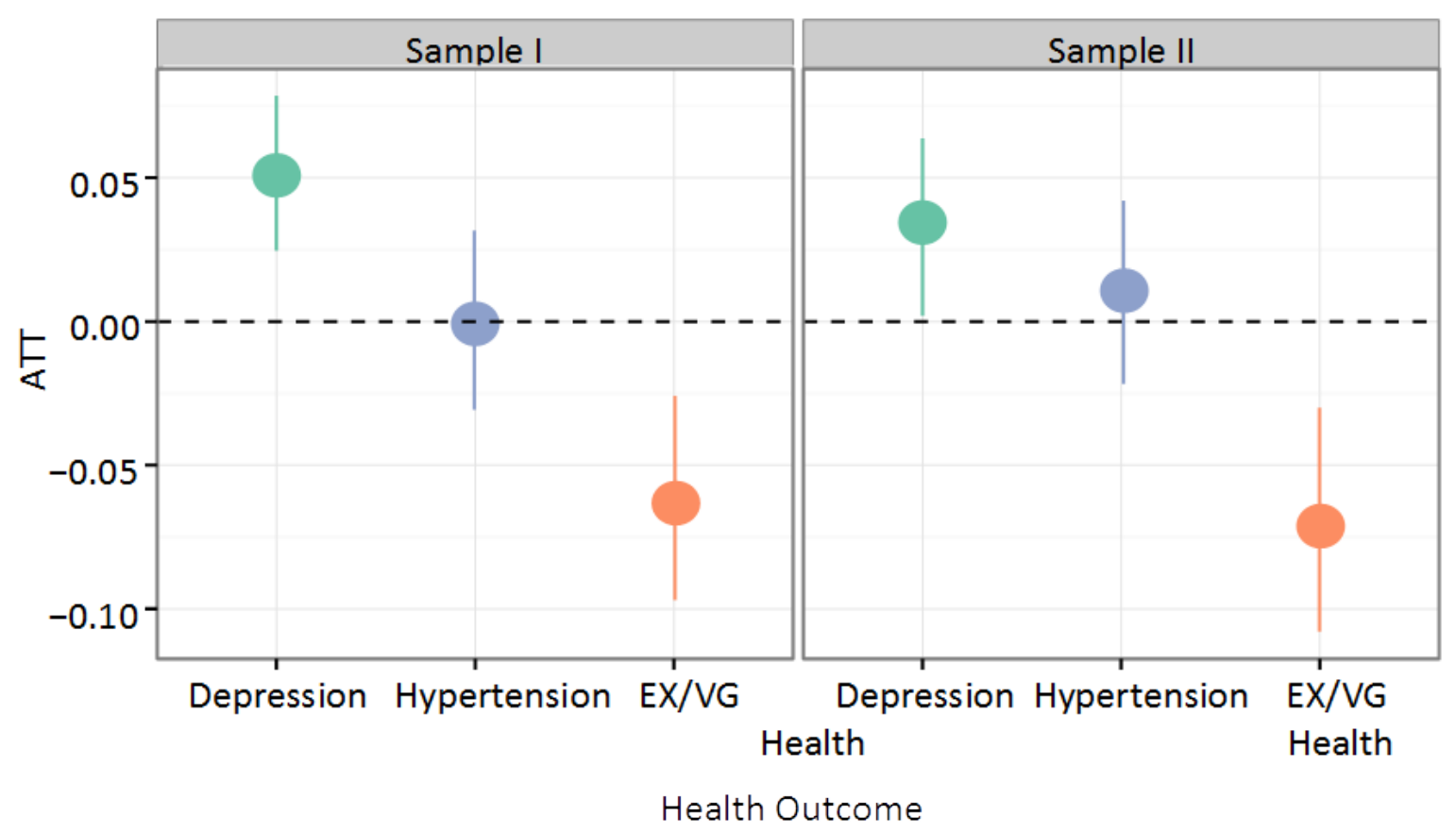

Figure 2: Estimated ATTs for each health outcome and each sample; $95 \%$ uncertainty intervals are marked.

Across analytical samples we see similar results. Net of the large set of confounders, the probability of being depressed would have been approximately $5 \%$ lower had individuals with a history of incarceration never been incarcerated. Note that this estimate is similar in magnitude to the difference in proportions given in figure 1 . That this health disadvantage persists in full after making individuals equivalent on all factors except prior incarceration, suggests that the elevated risk of depression among incarcerated individuals is largely a consequence of their incarceration. Similarly, having a prior history of incarceration appears to come with a $10 \%$ decrease in the probability of reporting excellent/very good health. This estimate is nearly half of what the difference in excellent/very good self-rated health was before accounting for confounders. This suggests that, while incarceration negatively affects general health status, a large portion of the association between health status and incarceration is simply a reflection of differences in factors that occur prior to incarceration.

In contrast to the previous two findings, our results show no adverse effects of incarceration on hypertension. The relationship between prior incarceration and hypertension represented in figure 1 dissipates after accounting for confounders, suggesting that those who were incarcerated before W4 would have been just as likely to experience hypertension had they never been incarcerated.

\section{Discussion}

In this paper, we expand the current understanding of how incarceration and health relate by examining the association between incarceration and health in the transition to adulthood. Understanding how incarceration impacts health during this period in the life course has been largely unexplored. Our results provide some support for the idea that incarceration during emerging adulthood is 
harmful to health. Taking account of a large number of features that occur prior to incarceration, experiences with incarceration during late adolescence and early adulthood appear to increase an individual's risk of depression, and negatively impact general health status. Given that health trajectories begin to solidify in young adulthood, these findings, which show incarceration effects early in adulthood, suggest that early confinement may have implications for health much later in the life course.

Our results, however, suggest that experiences with incarceration during this period have no discernible effect on risk of hypertension. The results for hypertension are surprising, given that earlier work suggests that incarceration significantly influences cardiovascular health (Binswanger, Krueger \& Steiner, 2009; Massoglia, 2008). It may be that Add Health respondents are simply not old enough to have manifested high blood pressure after exposure to stressors. Recent estimates indicate that approximately $10 \%$ of individuals aged between 18 and 45 years suffer from hypertension, compared to $40 \%$ of those between ages 45-64 (though Add Health reports substantially higher prevalence of hypertension among young adults than other national datasets do [such as the National Health and Nutrition Examination Survey]) (Keenan \& Rosendorf, 2011; Nguyen et al., 2011). In addition, young adults may be more resilient to stressors, and may be more likely to receive help from family members while confined, and after release. Since prior studies typically utilize samples of mid-age adults (such as the National Longitudinal Study of Youth 1979), this age difference may also account for the discrepancy in results. Our measure of incarceration includes both juvenile and adult confinement. Previous research indicates that these experiences differ (Loughran et al., 2010), and these differences may have different implications for health. Therefore, our unadjusted estimates of the association between confinement and health are likely to be somewhat conservative.

Our results suggest a number of directions for future work.
First, the relationship between incarceration during the transition to adulthood and health should continue to be explored by incorporating additional measures of mental and physical health as outcomes (including clinical measures of depression), and by empirically identifying the mechanisms that propagate this relationship. Our findings signal that incarceration is an important stressor that can have serious implications for life course outcomes even in the early stages of adulthood. Consequently, research should continue to increase our understanding of how incarceration in this emergent life stage matters for health.

Second, future researchers might use BART to examine other well-known consequences of incarceration (e.g. children's education outcomes, family dissolution, employment opportunities) (Lopoo \& Western, 2005; Turney \& Wildeman, 2013; Western, 2002). As with the association between incarceration and health, the associations among incarceration and many of these alternative outcomes are likely confounded by a large number of preexisting features. Therefore, using BART to isolate incarceration's influence on these outcomes could provide more exact insight into the impact that incarceration has on individuals' lives.

Third, future research should continue to make use of cutting-edge methods to examine incarceration's health effect. Though BART is useful in that it can coherently account for many confounding forces, it is limited in that it can only control for measured covariates. While we believe that our set of confounders is quite comprehensive, it is possible that we have not controlled for all of the features that jointly influence health and incarceration. As new methodologies that leverage observational data for causal inference become available, researchers should implement them, and compare their results with ours. A catalogue of results, produced via methodologies with varying strengths and weaknesses, would allow us to better quantify our confidence in estimates of incarceration's effect on health (e.g. if estimates of this quantity are similar across studies of varying approaches, we would be 
confident that our understanding of how incarceration and health relate is not a by-product of bias that comes from drawing causal inferences from observational data).

Fourth, investigating if (and how) the effect of incarceration on health varies across individuals is an important next step. Though data limitations kept our study from fully examining this point, incarceration is not a unidimensional or simple dichotomous variable. Indeed, the experience of incarceration can vary tremendously: while some incarcerated individuals are exposed to rather severe conditions (such as solitary confinement, over-crowded living spaces, or persistent physical abuse), others are confined in much less (overtly) harmful settings (e.g. low-security, rehabilitation-oriented facilities) (Kifer, Hemmens, \& Stohr, 2003; Smith, 2006; Wolff, Blitz, Shi, Siegel \& Bachman, 2007). This variation in incarceration experience may well lead to variation in incarceration's health effect (e.g. one might predict that individuals who have been exposed to long spells of solitary confinement while incarcerated might be more harmed than individuals who never experienced solitary confinement while detained). Additionally, a dose-response relationship (where the adverse health consequences of incarceration become more severe the longer an individual is incarcerated) may exist. So future research should focus on identifying the degree to which incarceration's health effect depends on the conditions under which an individual was incarcerated.
To this end, we believe that examining the association between juvenile incarceration and health in other countries would be productive. In the U.S. contact with the juvenile justice system does not necessarily serve to successfully rehabilitate or reintegrate individuals back into society. This may not be the case in other contexts, where incarceration may be a necessary, therapeutic family-level intervention. In these more rehabilitative contexts, incarceration's effect on health may prove to be less negative. As this is the case, the association between incarceration and health may vary across contexts, and should be explored in more depth (Andrews \& Bonta, 2010; Jones \& Newburn, 2005). Finally, a recent review of evidence by the National Academy of Sciences and the Institute of Medicine has determined that the U.S. population, on average, has lower life expectancies and higher rates of disease and injury compared to people in other high-income countries (Institute of Medicine, 2013). Moreover, this health disadvantage can be evidenced at all ages, and the high rates of illness and death along those younger than age 50 years is particularly troubling (Hummer \& Lawrence, 2015). The reasons for these troubling health disadvantages are multifactorial. However, it is likely that the high rates of incarceration for both youth and adults in the U.S. may also be driving some of these population differences. Future research should examine the important role incarceration may play as a driver of population health differences between the U.S. and other high-income countries, particularly at young ages.

\section{Acknowledgments}

This research uses data from Add Health, a program project directed by Kathleen Mullan Harris and designed by J. Richard Udry, Peter S. Bearman, and Kathleen Mullan Harris at the University of North Carolina at Chapel Hill, and funded by grant P01-HD31921 from the Eunice Kennedy Shriver National Institute of Child Health and Human Development, with cooperative funding from 23 other federal agencies and foundations. Special acknowledgment is due to Ronald R. Rindfuss and Barbara Entwisle for assistance in the original design. Information on how to obtain the Add Health data files is available on the Add Health website (http://www.cpc.unc.edu/addhealth). No direct support was received from grant P01-HD31921 for this analysis. We also thank the editor, and two anonymous reviewers for their helpful comments. 


\section{References}

Alwin, D., \& Wray, L. (2005). A life-span developmental perspective on social status and health. Journal of Gerontology: Series B, 60(2), S7-S14. https://doi.org/10.1093/geronb/60.Special_Issue_2.S7

Acharya, A., Blackwell, M., \& Sen, M. (2015). Detecting direct effects and assessing Alternative mechanisms. Under review. Accessed 15 March 2014 from http://j.mp/1Ee6Eaq.

Annie E. Casey Foundation. (2013). Reducing youth incarceration in the United States: KIDS COUNT data snapshot: youth incarceration in the United States. Accessed 14th March 2015 from http://tinyurl.com/ofv2f4u.

Andrews, D., \& Bonta, J. (2010). Rehabilitating criminal justice policy and practice. Psychology, Public Policy and Law, 16(1), 39-55. https://doi.org/10.1037/a0018362

Ben-Shlomo, Y., and Kuh, D. (2002). A life course approach to chronic epidemiology: conceptual models, empirical challenges, and interdisciplinary perspectives. International Journal of Epidemiology, 31, 286293. https://doi.org/10.1093/ije/31.2.285

Boardman, J., \& Alexander, K. (2011). Stress trajectories, health behaviors, and the mental health of black and white young adults. Social Science and Medicine, 72(10): 1659-1666. https://doi.org/10.1016/j.socscimed.2011.03.024

Binswanger, I., Krueger, P., \& Steiner, J. (2009). Prevalence of chronic medical conditions among jail and prison inmates in the United States compared with the general population. Journal of Epidemiology and Community Health, 63(11), 912-919. https://doi.org/10.1136/jech.2009.090662

Braveman, P., \& Gottlieb, L. (2014). The social determinants of health: it's time to consider the causes of the causes. Public Health Reports, 129(Suppl., 2), 19-31.

Cable, N. (2014). Life course approach in social epidemiology: an overview, application and future implications. Journal of Epidemiology, 24(5), 347-352. https://doi.org/10.2188/jea.JE20140045

Calhoun, D., Jones, D., Textor, S., Goff, D., Murphy, T., Toto, R., White, A., Cushman, C., White, W., Sica, S., Ferdinand, K., Giles, T., Falkner, B., \& Carey, R. (2009). Resistant hypertension: diagnosis, evaluation, and treatment: a scientific statement for high blood pressure research from the American Heart Association Professional Education Committee of the Council for High Blood Pressure Research. Hypertension, 51, 1403-1419. https://doi.org/10.1161/HYPERTENSIONAHA.108.189141

Chipman, H., George, E., \& McCulloch, R. (2010). BART: Bayesian Additive Regression Trees. Annals of Applied Statistics, 4, 266-298. https://doi.org/10.1214/09-AOAS285

Cutler, D., \& Lleras-Muney, A. (2006). Education and health: evaluating theories and evidence. NBER Working Paper 12352. National Bureau of Economic Research, Chicago, IL. https://doi.org/10.3386/w12352

Diamond, P., Wang, E., Holzer, C., Thomas, C., \& Cruse, A. (2001). The prevalence of mental illness in prison. Administration and Policy in Mental Health and Mental Health Services Research, 29(1), 21-40. https://doi.org/10.1023/A:1013164814732

Eaton, W., Muntaner, C., Smith, C., Tien, A., \& Ybarra, M. (2004). Center for Epidemiologic Studies Depression Scale: Review and revision (CESD and CESD-R). In M. Maruish (Ed.), The use of psychological testing for treatment planning and outcomes assessment. (pp. 363- 377). Mahwah, NJ: Lawrence Erlbaum.

Glaze, L., \& Parks, E., (2011). Correctional populations in the United States, 2011. U.S. Bureau of Justice Statistics. NCJ 239972.

Geller, A., \& Curtis, M. (2011). A sort of homecoming: incarceration and the housing security of urban men. Social Science Research, 40(4), 1196-1213. https://doi.org/10.1016/j.ssresearch.2011.03.008 
Gelman, A., \& Hill, J. (2007). Data analysis using regression and multilevel/hierarchical models. New York: Cambridge University Press.

Golzari, M., Hunt, J., \& Anoshiravani, A. (2006). The health status of youth in juvenile detention facilities. Journal of Adolescent Health, 38, 776-782. https://doi.org/10.1016/j.jadohealth.2005.06.008

Goldstein, A., Kapelner, A., Bleich, J., \& Pitkin, E. (2014). Peeking inside the black box: visualizing statistical learning with plots of individual conditional expectation. Journal of Computational and Graphical Statistics, 24(1),

Green, D., \& Kern, H. (2012). Modeling heterogeneous treatment effects in survey experiments with Bayesian Additive Regression Trees. Public Opinion Quarterly, 76(3), 491-511.

Haas, S. (2007). The long-term effects of poor childhood health: an assessment and application of retrospective reports. Demography, 44(1), 113-135. https://doi.org/10.1353/dem.2007.0003

Hale, D., Bevilacqua, L., \& Viner, R. (2015). Adolescent health and adult education and employment: a systematic review. Pediatrics, 126(1), 128-140. https://doi.org/10.1542/peds.2014-2105

Harris, K.M., Halpern, C., Whitsel, E., Hussey, J., Tabor, J., Entzel. P., \& Udry, J. (2009). The National Longitudinal Study of Adolescent Health: research design. Accessed 14th March 2015 from http://www.cpc.unc.edu/projects/addhealth/design

Hawe, P., \& Shiell., P. (2000). Social capital and health promotion: a review. Social Science and Medicine. 51(6), 871-85. https://doi.org/10.1016/S0277-9536(00)00067-8

Hazel, N. (2008). Cross-national comparisons of youth justice. London: Youth Justice Board. Accessed 14th March 2015 from http://tinyurl.com/o348r3k.

Hill, J. (2011). Bayesian nonparametric modeling for causal inference. Journal of Computational and Graphical Statistics, 20(1), 217-240. https://doi.org/10.1198/jcgs.2010.08162

Hill, J., Weiss, C., \& Zhai, F. (2011). Challenges with propensity score strategies in a high-dimensional setting and a potential alternative. Multivariate Behavioral Research, 46(3), 477-513. https://doi.org/10.1080/00273171.2011.570161

Hirschi, T. (1969). Causes of delinquency. University of California Press.

Ho, D., Imai, K., King, G., \& Stuart, E. (2007). Matching as nonparametric preprocessing for reducing model dependence in parametric causal inference. Political Analysis, 15, 199-236. https://doi.org/10.1093/pan/mpl013

Holzer, H., Raphael, S., \& Stoll, M. (2004). How willing are employers to hire ex-offenders? Focus, 23(2), 40-43.

Hummer, R., \& Lawrence, E. (2015). Racial/ethnic differences in early life mortality in the United States. Poster presented at the annual meeting of the Southern Demographic Association, San Antonio, Texas.

Institute of Medicine. (2013). U.S. health in international perspective: shorter lives, poorer health. Accessed November 2015 from http://tinyurl.com/oe8ahzu.

James, G., Witten, D., Hastie, T., \& Tibshirani, R. (2015). An introduction to statistical learning: with applications in $R$. New York: Springer.

Jones, T., \& Newburn T. (2005). Comparative criminal justice policy-making in the United States and the United Kingdom. The British Journal of Criminology, 45(1), 58-80. https://doi.org/10.1093/bjc/azh067

Kapelner, A. \& Bleich, A. (2016). bartMachine: Machine Learning with Bayesian Additive Regression Trees. Journal of Statistical Software, 70(4), 1-40. https://doi.org/10.18637/jss.v070.i04

Keenan, N., \& Rosendorf, K. (2011). Prevalence of hypertension and controlled hypertension - United States, 2005-2008. MMWR Surveillance Summaries, 60(S): 94-97.

Kifer, M., Hemmens, C., \& Stohr, M. K. (2003). The goals of corrections: perspectives from the line. Criminal Justice Review, 28(1), 47-49. https://doi.org/10.1177/073401680302800104 
Lee, H., Wildeman, C., Wang, E., Matusko, N., \& Jackson, J. (2014). A heavy burden: the cardiovascular health consequences of having a family member incarcerated. American Journal of Public Health, 104(3), 421427. https://doi.org/10.2105/AJPH.2013.301504

Loughran, T. A., Mulvey, E. P., Schubert, C. A., Chassin, L. A., Steinberg, L., Piquero, A. R., Cota-Robles, S., Fagan J., Cauffman E., \& Losoya, S. H. (2010). Differential effects of adult court transfer on juvenile offender recidivism. Law and Human Behavior, 34(6), 476-488. https://doi.org/10.1007/s10979-009-9210-z

Lopoo, L., \& Western, B. (2005). Incarceration and the formation and stability of marital unions. Journal of Marriage and Family, 67(3), 721-734. https://doi.org/10.1111/j.1741-3737.2005.00165.x

Mandal, M., Edelstein, B. L., Ma, S., \& Minkovitz, C. S. (2013). Changes in children's oral health status and receipt of preventive dental visits, United States, 2003-2011/2012. Preventing Chronic Disease, 10, E204. https://doi.org/10.5888/pcd10.130187

Maruschak, L. (2010). HIV in Prisons, 2001-2010. NCJ 238877. Washington D.C.: U.S. Bureau of Justice Statistics.

Massoglia, M. (2008). Incarceration as exposure: the prison, infectious disease, and other stress-related illnesses. Journal of Health and Social Behavior, 49(1), 56-71. https://doi.org/10.1177/002214650804900105

Massoglia, M., \& Pridemore, W. (2015). Incarceration and health. Annual Review of Sociology, 41, 291-310. https://doi.org/10.1146/annurev-soc-073014-112326

Mirowsky, J., \& Ross, C. (2003). Education, social status, and health: social institutions and social change. New York: Aldine de Gruyter.

Nguyen, Q., Tabor, J., Entzel, P., Lau, Y., Suchindran, C., Hussey, J., Halpern, C., Harris, K., \& Whitsel, E. (2011). Discordance in national estimates of hypertension among young adults. Epidemiology, 22(4), 532-541. https://doi.org/10.1097/EDE.0b013e31821c79d2

Pettit, B., \& Western, B. (2004). Mass imprisonment and the life course: race and class inequality in U.S. incarceration. American Sociological Review, 69(2), 151-169. https://doi.org/10.1177/000312240406900201

Perreira, K. M., Deeb-Sossa, N., Harris, K. M., \& Bollen, K. A. (2005). What are we measuring? An evaluation of the CES-D across race/ethnicity and immigrant generation. Social Forces 83(4), 1567-1601. https://doi.org/10.1353/sof.2005.0077

Pervanidou, P., \& Chrousos, G. (2012). Metabolic consequences of stress during childhood and adolescence. Metabolism, 61(5), 611-619. https://doi.org/10.1016/j.metabol.2011.10.005

Phelan, J., Link, B., \& Tehranifar, P. (2010). Social conditions as fundamental causes of health inequalities: theory, evidence, and policy implications. Journal of Health and Social Behavior, 51(1): S28-S40. https://doi.org/10.1177/0022146510383498

Phelan, J., \& Link, B. (2015). Is racism a fundamental cause of inequalities in health? Annual Review of Sociology, 41, 311-330. https://doi.org/10.1146/annurev-soc-073014-112305

Porter, L. (2014). Incarceration and post-release health behavior. Journal of Health and Social Behavior, 55(2), 234-249. https://doi.org/10.1177/0022146514531438

Robards, J., Evandrou, M., Falkingham, J., \& Vlachantoni, A. (2012). Marital status, health and morality. Maturitas, 73(4): 295-299. https://doi.org/10.1016/j.maturitas.2012.08.007

Roettger, M., \& Boardman, J. (2012). Parental incarceration and gender-based risks for increased body mass index: evidence from the National Longitudinal Study of Adolescent Health in the United States. American Journal of Epidemiology, 175(7), 636-644. https://doi.org/10.1093/aje/kwr409

Rose, D. R., \& Clear, T. R. (1998). Incarceration, social capital and crime: implications for social disorganization theory. Criminology, 36(3), 441-480. https://doi.org/10.1111/j.1745-9125.1998.tb01255.x 
Settersten Jr, R., Furstenberg, F., \& Rumbaut, R. (2005). On the frontier of adulthood: theory, research and public policy. Chicago: University of Chicago Press. https://doi.org/10.7208/chicago/9780226748924.001.0001

Schnittker, J., \& Bacak, V. (2013). A mark of disgrace or a badge of honor? Subjective status among former inmates. Social Problems, 60(2), 234-254. https://doi.org/10.1525/sp.2013.60.2.234

Schnittker, J., \& John, A. (2007). Enduring stigma: the long-term effects of incarceration on health. Journal of Health and Social Behavior, 48(2), 115-130. https://doi.org/10.1177/002214650704800202

Schnittker, J., Massoglia, M., \& Uggen, C. (2012). Out and down: incarceration and psychiatric disorders. Journal of Health and Social Behavior, 53(4), 448-464. https://doi.org/10.1177/0022146512453928

Smith, P. S. (2006). The effects of solitary confinement on prison inmates: a brief history and review of the literature. Crime and Justice, 34(1), 441-528. https://doi.org/10.1086/500626

Turney, K., \& Wildeman, C. (2013). Redefining relationships: explaining the countervailing consequences of parental incarceration for parenting. American Sociological Review, 78(6), 949-979. https://doi.org/10.1177/0003122413505589

Walmsley, R. (2013). World prison population list, $10^{\text {th }}$ edition. International Centre for Prison Studies. Accessed September 2015 from http://tinyurl.com/qc9ktdc.

Western, B. (2002). The impact of incarceration on wage mobility and inequality. American Sociological Review, 67, 526-546. https://doi.org/10.2307/3088944

Western, B. (2006). Punishment and inequality in America. New York: Russell Sage.

Western, B., \& Pettit, B. (2010). Incarceration and social inequality. Daedalus, 139(3), 8-19. https://doi.org/10.1162/DAED_a_00019

Western, B., Kling, J. R., \& Weiman, D. F. (2001). The labor market consequences of incarceration. Crime \& Delinquency, 47(3), 410-427. https://doi.org/10.1177/0011128701047003007

Williams, D., Mohammed, S., Leavell, J., \& Collins, C. (2010). Race, socioeconomic status and health: complexities, ongoing challenges and research opportunities. Annals of New York Academy of Sciences, 1186, 69-101. https://doi.org/10.1111/j.1749-6632.2009.05339.x

Wildeman, C., Schnittker, J., \& Turney, K. (2012). Despair by association? The mental health of mothers with children by recently incarcerated fathers. American Sociological Review 77(2), 216-243.

Wolff, N., Blitz, C., Shi, J., Siegel, J., \& Bachman, R. (2007). Physical violence inside prison: rates of victimization. Criminal Justice and Behavior, 34, 588-599. https://doi.org/10.1177/0093854806296830

\section{Endnotes}

i Post-treatment bias is, in part, a form of selection bias that occurs when conditioning on variables that are affected by the treatment of interest (i.e. incarceration, in our case), and affect the outcome of interest (i.e. health) (Acharya et al., 2015). This form of bias can be extremely conservative or anti-conservative, and can leave estimates with no substantive, causal interpretation. So avoiding this form of bias is paramount to our efforts. For a more detailed overview of post-treatment bias see Acharya et al. (2015).

ii Note that these additional 36 variables allow for Sample II's estimates to be at least as accurate as Sample I's estimates. For example, self-rated health at W1 is controlled for in Sample I and Sample II, where self-rated health at W3 is controlled for only in Sample II (given that Sample I individuals had already experienced 
incarceration by the time their W3 health was recorded). If W3 self-rated health were not controlled for in Sample II, but W1 self-rated health was still controlled for in Sample I, our estimates for Sample II would not account for an individual's general health status immediately before they were incarcerated, but our Sample I estimates would. This may lead to more biased results for Sample II.

iii Because the size of the confounder set is so large, a rationale explaining each variable's inclusion is beyond the scope of this paper.

iv While hypertension is not often analyzed as an outcome in young adult populations, a remarkable number of individuals in Add Health are coded as having hypertension (approximately 20\% of individuals at W4) (Nguyen et al., 2011). Because the proportion of individuals with hypertension is quite sizable in these data, and because (as will be discussed later) the prevalence of hypertension is elevated among the observed groups of individuals with a history of incarceration, we believe that it is useful to analyze hypertension as an outcome in this manuscript.

$v$ These concerns manifest themselves in our analysis: logistic regression models of our health outcomes (in which all covariates were specified in a simple, additive fashion) either failed to converge, or failed to identify some parameter estimates when the full set of confounders were included as covariates.

vi For instance, while prior research strongly suggests that income, educational attainment, and race are confounders of incarceration's health effect (e.g. Mirowsky \& Ross, 2003; Western \& Pettit, 2010), it provides little guidance on how these factors ought to enter into a model predicting health as a function of incarceration. Questions such as, "does incarceration's health effect vary by pre-incarceration income" (i.e. does the model need an interaction between incarceration and income?), or "does the degree to which incarceration's negative health effect vary by education vary by race" (i.e. does the model need a three-way interaction between incarceration, race, and education) are not covered in detail in existing work.

vii Because BART is a nonparametric, "black-box" machine-learning approach, we are not provided parameter estimates for our models (Goldstein, Kapelner, Bleich, \& Pitkin, 2014; Green \& Kern, 2012). To establish estimates of what variables matter and to gain a (rough) sense of the structure of each BART model, we calculate each variable's inclusion proportion. This quantity measures the number of times a specific variable was used in BART's tree models, divided by the total number of variables used in all of the BART's tree models (Chipman et al., 2010; Kaplener \& Bleich,2016) The more often a variable is used in predicting the response, the higher its inclusion proportion will be.

Plots of the top 20 variables by inclusion proportion (for each model) are included in the supplemental materials. Note that, for the Sample I and Sample II self-rated health and depression models, socialpsychological features (e.g. assessment of own abilities, frequency of "feeling down", or assessment of relationship with their peers) have relatively high variable inclusion proportions. Also note that factors measuring engagement in deviant behaviour (e.g. counts of times that an individual has stolen something, has 'graffitied', and (in Sample II) was a victim of a violent crime) have relatively large inclusion proportions in our models of hypertension. 